\title{
Direct Synthesis of Multiband Bandpass Filters With Generalized Frequency Transformation Methods
}

\author{
Yi Wu ${ }^{\circledR}$, Student Member, IEEE, Erwan Fourn ${ }^{\circledR}$, Member, IEEE, Philippe Besnier ${ }^{\circledR}$, Senior Member, IEEE, \\ and Cédric Quendo ${ }^{(\mathbb{D}}$, Senior Member, IEEE
}

\begin{abstract}
This article presents a new generalized direct synthesis method to design multiband bandpass filters (MBPFs). Based on the proposed frequency transformation technique, it can be used to synthesize an arbitrary number of bandpass filters starting from a low-pass prototype filter. This synthesis allows determining analytically all the resonant angular frequencies and slope parameters of all the bandpass filters from the low and high cut-off angular frequencies used as initial specifications whatever the number of bands. To validate our method, triple-, quad-, and quint-band third-order Chebyshev bandpass filters are designed and implemented in microstrip technologies. Very good agreements were achieved between simulation responses and measurements.
\end{abstract}

Index Terms-Frequency transformation methods, microstrip technology, multiband bandpass filter (MBPF), quad-band filter, quint-band filter, triple-band filter.

\section{INTRODUCTION}

$\mathbf{F}$ ILTERS are key components in radio frequency transceivers in handing multiband operations. Their integration is challenging with regard to requirements. In new terminal generations providing several communication standards simultaneously, and especially in mobile terminals, embedded systems, or satellites, the footprint of the filtering parts needs to be reduced in order to meet mass and volume requirements of the whole system. Multiband bandpass filters (MBPFs) indeed then appear as an attractive solution to select multiple bands with a relatively compact circuit size. Thus, [1], [2] present some examples of MBPFs to simplify the design of communication system for satellite applications. Many researchers pay great attention on MBPFs leading to a large amount of circuit topologies implemented in different technologies such

Manuscript received October 20, 2020; revised March 9, 2021 and April 13, 2021; accepted May 11, 2021. This work was supported in part by the European Union through the European Regional Development Fund (ERDF) and in part by the French Region of Brittany, Ministry of Higher Education and Research, Rennes Métropole and Conseil Départemental 35, through the Contrat Plan État Région (CPER) Project SOPHIE/STIC \& Ondes. (Corresponding author: $\mathrm{Yi}$ Wu.)

Yi Wu, Erwan Fourn, and Philippe Besnier are with Univ Rennes, INSA Rennes, CNRS, IETR-UMR 6164, F-35000 Rennes, France (e-mail: yi.wu@insa-rennes.fr; erwan.fourn@insa-rennes.fr; philippe.besnier@ insa-rennes.fr).

Cédric Quendo is with the Lab-STICC Laboratory, University of Brest, 29238 Brest, France (e-mail: cedric.quendo@univ-brest.fr).

Color versions of one or more figures in this article are available at https://doi.org/10.1109/TMTT.2021.3086835.

Digital Object Identifier 10.1109/TMTT.2021.3086835 as microstrip [3]-[7], waveguide [8], or substrate integrated waveguide (SIW) [9]-[11].

More specifically, the synthesis of MBPFs, whatever the implementation technology, can be done through numerous methods. One of the most popular ones consists in deriving coupling matrix to achieve multiband responses [12]-[16]. Actually, this method is based on the optimization of the whole coupling matrix. High quality of initial values and good optimization algorithms (such as genetic algorithm or sequential quadratic programming) are needed to achieve satisfying results. Such methods may be considered inefficient in some cases because the convergence is not always guaranteed and the final topology is difficult to control [16], [17]. A second popular method to design MBPF is based on stepped-impedance resonators (SIRs) with two main concepts. The first one makes use of multisection SIRs with different resonant frequencies [18]-[24]. These resonators can have different shapes such as ring or stub-loaded shapes or consist of an assembling of quarter- or half-wavelength lines. For such filters, each resonator can resonate at the center frequencies of at least two bands. By coupling these resonators together, one can realize multibands responses. However, MBPFs' design, especially beyond triple-bands, is still challenging because it is really difficult to simultaneously satisfy all the desired requirements for all bandpass, including resonant frequencies, bandwidths, and so on. A third method consists in using dual behavior resonator (DBR) filter [25], [26]. A DBR is based on the parallel association of $N$ band-stop resonators, which can create $N$ transmission zeros to form $N-1$ bands. Based on this method, dual- and triple-band bandpass filters have been successfully synthesized and implemented. However, for this type of filter, it is hardly possible to achieve more bandpass, because of the harmonic frequencies, and high-order filters because slope parameters are not taken into account into the synthesis.

Another MBPFs' synthesis technique is based on frequency transformation methods. The first article was presented in [27] in the case of a dual-band filter. The main idea of this article is to map the lower and upper cut-off angular frequencies of each bandpass to the low-pass prototype ones (positive and negative). The dual-band resonator obtained using this transformation then consists in the association of a bandpass resonator and a band-stop one, the latter creating a transmission

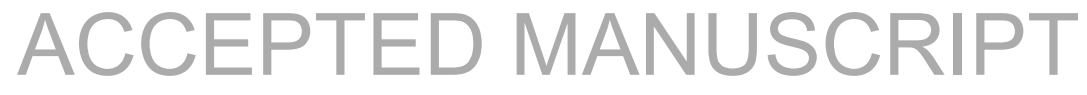




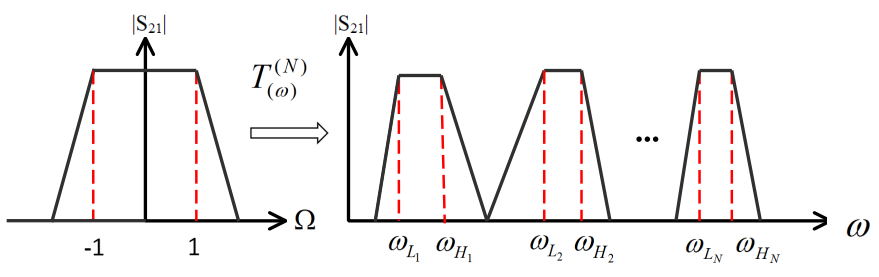

Fig. 1. From a low-pass prototype to an $N$-band bandpass filter.

zero in the bandpass of the first to obtain the two bands. Similar techniques were used to design dual- and triple-band bandpass filters in [28] and [29] with different implementation topologies and technologies. In [30], the authors also applied the frequency transformation approach to design triple-band bandpass filter. Additional transmission zeros were realized before each bandpass using optimized multimode resonators. However, the optimization-based multimode resonators are difficult to be implemented for filters more than triple bandpass. A generalized synthesis method was proposed in [31]. In this article, dual- and triple-band cases were presented to validate the synthesis theory. This approach is based on coupling matrices and frequency-invariant susceptances. However, practical implementation of multiband filters with more than three bands seems rather difficult.

In this article, a general analytical method to design MBPFs with an arbitrary number of bands based on frequency transformation technique is presented. It is a direct generalization of the solution presented in [27] and differs from [31] by the use of the slope parameters instead of coupling matrices, leading to a relative greater simplicity and freedom in terms of design and implementation. The design of high-order MBPFs with an important number of bands is then relatively easy unlike most of the solutions discussed above. To demonstrate the effectiveness of this approach, several synthesis examples are also shown. The article is organized as follows: Section II presents the generalized frequency transformation technique in its mathematical aspects. Section III shows the ideal implementation in $L C$ element structure and Section IV presents three examples of MBPFs implemented in microstrip technology: A triple-band filter, a quad-band filter, and a quint-band filter. The last section concludes this article.

\section{Generalized Frequency Transformation TECHNiQue FOR Multiband Bandpass Filters}

The transformation from a classical low-pass prototype to an $N$-band bandpass filter is schematically presented in Fig. 1. The proposed frequency transformation function is a generalization of the one proposed in [27] in the dual-band case and can be written as follows:

$$
\begin{aligned}
\Omega & =T^{(N)}(\omega) \\
& =b_{0}\left(\frac{\omega}{\omega_{0}}-\frac{\omega_{0}}{\omega}\right)-\sum_{k=1}^{N-1} \frac{1}{b_{k}\left(\frac{\omega}{\omega_{k}}-\frac{\omega_{k}}{\omega}\right)}
\end{aligned}
$$

where $\Omega$ is the normalized angular frequency (associated with the normalized low-pass filter). $T^{(N)}(\omega)$ is the transformation function, $N$ the number of bands of the MBPFs, and $\omega$ the de-normalized angular frequency (associated with the MBPFs), $\omega_{0}$ and $b_{0}$ are the resonant angular frequency and the susceptance slope parameter of the wide bandpass resonator, respectively, $\omega_{k}$ and $b_{k}(k \in[1 ; N-1])$ are the ones of the stopband resonators.

The goal of the synthesis process is to link the $2 \mathrm{~N}$ output parameters of the transformation function (the resonant angular frequencies $\omega_{k}$ and the slope parameters $b_{k}, k \in[1 ; N-1]$ ) to the input ones, i.e., the low and high cut-off angular frequencies of the $N$ bands $\left(\omega_{L_{i}}\right.$ and $\left.\omega_{H_{i}}, i \in[1 ; N]\right)$ as defined in Fig. 1.

First, let us denote

$$
U^{(N)}(\omega)=T^{(N)}(\omega)-1
$$

Substituting equation (1) for $T^{(N)}(\omega)$ in (2) leads to a new expression of $U^{(N)}(\omega)$ consisting in a ratio of two polynomials

$$
U^{(N)}(\omega)=\frac{N(\omega)}{D(\omega)}=\frac{\omega^{2 N}+\sum_{p=0}^{2 N-1} n_{p} \omega^{p}}{\sum_{q=0}^{2 N-1} d_{q} \omega^{q}}
$$

where $d_{q}$ is equal to 0 when $q$ is even and $-n_{q}$ when $q$ is odd. A first expression of the $2 N n_{p}(p \in[0 ; 2 N-1])$ coefficients is then obtained as functions of the output parameters as detailed below.

We now assume that the lower cut-off angular frequencies $\omega_{L_{i}}$ and the upper cut-off angular frequencies $\omega_{H_{i}}(i \in[1 ; N])$ are mapped to -1 and +1 in the $\Omega$ normalized domain, respectively. As $T^{(N)}(\omega)$ is an odd function, we then have

$$
T^{(N)}\left(-\omega_{L_{i}}\right)=T^{(N)}\left(\omega_{H_{i}}\right)=1 \quad i \in[1 ; N] .
$$

Therefore, the $N$ high cut-off angular frequencies and the opposite of the $N$ lower cut-off ones are the $2 N$ roots of $U^{(N)}(\omega)$. A second expression of the $n_{p}$ coefficients is thus obtained as functions of the input parameters this time.

So, $2 N$ equations (one for each $n_{p}$ coefficient) link the $N$ resonant angular frequencies, the $\omega_{k}$, and the $N$ slope parameters, $b_{k}((k \in[0 ; N-1]))$, to the $N$ low cut-off angular frequencies, $\omega_{L_{i}}$, and the $N$ high cut-off ones, $\omega_{H_{i}}$ $(i \in[1 ; N])$. As presented below, an analytical expression can then be obtained for each output parameters.

Section II-A-II-C present the general expressions of the $n_{p}$ coefficients $(p \in[0 ; 2 N-1])$ according to the cut-off angular frequencies (see Section II-B), on the one hand, and according to the resonant angular frequencies and slope parameters (see Section II-C), on the other hand. To simplify the writing of these equations and make the understanding easier, we first introduce specific mathematical expressions in Section II-A.

\section{A. Specific Mathematical Operators}

Let $\mathcal{Z}^{(r \rightarrow s)}$ be a set of real numbers $z_{i}$ with $i \in[r ; s](r \in \mathbb{N}$ and $s \in \mathbb{N}^{\star}$ )

$$
\mathcal{Z}^{(r \rightarrow s)}=\left\{z_{i}\right\}_{i=r}^{s} .
$$

We then denote $\mathcal{Z}_{\{j\}}^{(r \rightarrow s)}$ the same set of real numbers $z_{i}$ with $i \neq j((i ; j) \in[r ; s])$

$$
\begin{aligned}
\mathcal{Z}_{\{j\}}^{(r \rightarrow s)} & =\left\{z_{i}\right\}_{i=r}^{s} \backslash\left\{z_{j}\right\} \\
& =\left\{z_{r}, z_{r+1}, \ldots, z_{j-1}, z_{j+1}, \ldots, z_{s-1}, z_{s}\right\} .
\end{aligned}
$$


Let $\mathcal{P}_{a}\left(\mathcal{Z}^{(r \rightarrow s)}\right)$ be the sum of all the different products of $a$ elements of $\mathcal{Z}^{(r \rightarrow s)}$. $\mathcal{P}_{a}\left(\mathcal{Z}^{(r \rightarrow s)}\right)$ is thus a sum of $\left(\begin{array}{c}s-r+1 \\ a\end{array}\right)$ terms, each of them being a product of $a$ elements of $\mathcal{Z}^{(r \rightarrow s)}$. For instance

$$
\begin{aligned}
& \mathcal{P}_{1}\left(\mathcal{Z}^{(1 \rightarrow 3)}\right)=z_{1}+z_{2}+z_{3} \\
& \mathcal{P}_{2}\left(\mathcal{Z}^{(1 \rightarrow 3)}\right)=z_{1} z_{2}+z_{1} z_{3}+z_{2} z_{3} \\
& \mathcal{P}_{3}\left(\mathcal{Z}^{(1 \rightarrow 3)}\right)=z_{1} z_{2} z_{3}
\end{aligned}
$$

and

$$
\mathcal{P}_{2}\left(\mathcal{Z}_{\{3\}}^{(1 \rightarrow 4)}\right)=z_{1} z_{2}+z_{1} z_{4}+z_{2} z_{4}
$$

Finally, we denote

$$
\mathcal{P}_{0}\left(\mathcal{Z}^{(r \rightarrow s)}\right)=1
$$

\section{B. Expression of the $n_{p} C$ Coefficients as Functions of the Cut-Off Angular Frequencies}

In this section, an expression of the $2 N n_{p}$ coefficients used in (3) is obtained as functions of the frequency transformation input parameters, i.e., the cut-off angular frequencies of the $N$ bands. We use here the fact that the $N$ high cut-off angular frequencies $\omega_{H_{i}}$ and the opposite of the low cut-off ones $\omega_{L_{i}}$, with $i \in[1 ; N]$, are the zeros of $U^{(N)}(\omega)$. We thus obtain a first system of $2 N$ equations (one for each cut-off angular frequency) with $2 N$ unknowns $\left(n_{p}, p \in[0 ; 2 N-1]\right)$.

According to the expressions presented in Section II-A, we denote $\mathcal{L}^{(1 \rightarrow N)}=\left\{\omega_{L_{i}}\right\}$ and $\mathcal{H}^{(1 \rightarrow N)}=\left\{\omega_{H_{i}}\right\}, i \in[1 ; N]$. Solving this first system leads to

$$
n_{p}=\sum_{r=0}^{p}(-1)^{N-r} \mathcal{P}_{N-r}\left(\mathcal{H}^{(1 \rightarrow N)}\right) \mathcal{P}_{N+r-p}\left(\mathcal{L}^{(1 \rightarrow N)}\right)
$$

for $p \in[0 ; N-1]$

$$
=\sum_{r=p}^{2 N}(-1)^{2 N-r} \mathcal{P}_{2 N-r}\left(\mathcal{H}^{(1 \rightarrow N)}\right) \mathcal{P}_{r-p}\left(\mathcal{L}^{(1 \rightarrow N)}\right)
$$

for $p \in[N ; 2 N-1]$.

\section{Expression of the $n_{p}$ Coefficients as Functions of the Resonant Angular Frequencies and the Susceptance Slope Parameters}

We now express the $2 N n_{p}$ coefficients as functions of the frequency transformation output parameters, i.e., the resonant angular frequency, $\omega_{0}$, and the slope parameter, $b_{0}$, of the wide bandpass resonator, and the parameters $\omega_{k}$ and $b_{k}(k \in$ $[1 ; N-1])$. We recall that these parameters are the resonant angular frequencies and the slope parameters of the stopband resonators, respectively. This is based on the introduction of the expression of $T^{(N)}(\omega)$ from (1) in (2), which allowed us to obtain the expression of $U^{(N)}(\omega)$ given in (3). We thus obtain a second system of $2 N$ equations giving each $n_{p}$ coefficient as a function of the output parameters.

Here, we denote $\mathcal{W}^{(0 \rightarrow N-1)}=\left\{\omega_{k}^{2}\right\}$ the set of all the resonant angular frequency squares and $\mathcal{W}^{(1 \rightarrow N-1)}=\left\{\omega_{k}^{2}\right\}$ the set of the resonant angular frequency squares of the stopband resonators only. We distinguish three cases for $p=0, p$ odd, and $p$ even, $p \in[0 ; 2 N-1]$.

1) For $p=0$, we have

$$
\begin{aligned}
n_{0} & =(-1)^{N} \mathcal{P}_{N}\left(\mathcal{W}^{(0 \rightarrow N-1)}\right) \\
& =(-1)^{N} \prod_{r=0}^{N-1} \omega_{r}^{2} .
\end{aligned}
$$

2) For $p$ odd, we denote $p=2 q-1, q \in[1 ; N]$. We then have

$$
\begin{aligned}
n_{p} & =n_{2 q-1} \\
& =(-1)^{N-q+1} \frac{\omega_{0}}{b_{0}} \mathcal{P}_{N-q}\left(\mathcal{W}^{(1 \rightarrow N-1)}\right) .
\end{aligned}
$$

3) For $p$ even, we denote $p=2 q, q \in[1 ; N-1]$, and then we obtain

$$
\begin{aligned}
n_{p}=n_{2 q} & \\
=(-1)^{N-q}\{ & \mathcal{P}_{N-q}\left(\mathcal{W}^{(0 \rightarrow N-1)}\right) \\
& \left.\quad+\frac{\omega_{0}}{b_{0}} \sum_{k=1}^{N-1}\left[\frac{\omega_{k}}{b_{k}} \mathcal{P}_{N-q-1}\left(\mathcal{W}_{\{k\}}^{(1 \rightarrow N-1)}\right)\right]\right\} .
\end{aligned}
$$

\section{Expression of the Resonance Angular Frequencies and} Susceptance Slope Parameters

Equations (9) to (11) give an expression of the $2 N n_{p}$ as functions of the output parameters of the frequency transformation technique (i.e., $\omega_{k}$ and $b_{k}, k \in[0 ; N-1]$ ). In a practical way, we have to reverse this equation system in order to express the output parameters as functions of the $2 \mathrm{~N}$ $n_{p}$ coefficients, these latter being calculated from the input parameters $\left(\omega_{L_{i}}\right.$ and $\left.\omega_{H_{i}}(i \in[1 ; N])\right)$ using $(8)$.

First, using the expressions of $n_{0}, n_{1}$, and $n_{2 N-1}$ and whatever the number of bands $N$, we can easily demonstrate that the resonant frequency $\omega_{0}$ and the slope parameter $b_{0}$ of the wide bandpass filter can be written as

$$
\begin{aligned}
& \omega_{0}=\sqrt{-\frac{n_{0} n_{2 N-1}}{n_{1}}} \\
& b_{0}=\sqrt{-\frac{n_{0}}{n_{1} n_{2 N-1}}} .
\end{aligned}
$$

Using (10) for all the odd value of $p$, we can also demonstrate (see Appendix A) that the resonant angular frequencies of the band-stop resonators $\left.\left(\omega_{k}, k \in[1 ; N-1]\right)\right)$ are the $N-1$ positive solutions of (14)

$$
\sum_{r=0}^{N-1} n_{2 r+1} \omega_{k}^{2 r}=0
$$

Once the resonant angular frequencies of the band-stop resonators are determined using (14), the remaining unknowns are the slope parameter coefficients $b_{k}, k \in[1 ; N-1]$ in (11). This equation can also be rewritten as follows:

$$
\begin{aligned}
\sum_{k=1}^{N-1}\left[\frac{\omega_{k}}{b_{k}} \mathcal{P}_{N-q-1}\left(\mathcal{W}_{\{k\}}^{(1 \rightarrow N-1)}\right)\right] & \\
= & \frac{\mathcal{P}_{N-q}\left(\mathcal{W}^{(0 \rightarrow N-1)}\right)-(-1)^{N-q} n_{2 q}}{n_{2 N-1}}
\end{aligned}
$$


for $q \in[1 ; N-1]$. Using (15) for each value of $q$, we obtain an $N-1$ linear equation system with $(N-1)$ unknowns (i.e., each $b_{k}$ or more precisely $1 / b_{k}$ ). This system can be written as follows:

$$
X_{N-1, N-1} B_{N-1}=A_{N-1}
$$

where $X_{N-1, N-1}$ is the $(N-1 \times N-1)$ matrix of $x_{q, k}$ coefficients with

$$
x_{q, k}=\omega_{k} \mathcal{P}_{N-q-1}\left(\mathcal{W}_{\{k\}}^{(1 \rightarrow N-1)}\right) .
$$

$B_{N-1}$ is the vector of the $N-1$ unknowns

$$
B_{N-1}=\left[\frac{1}{b_{1}}, \frac{1}{b_{2}}, \ldots, \frac{1}{b_{N-1}}\right]^{t}
$$

and $A_{N-1}$ is the vector of already known coefficients $a_{q}^{(N)}$

$$
A_{N-1}=\left[a_{1}, a_{2}, \ldots, a_{N-1}\right]^{t}
$$

with

$$
a_{q}=\frac{\mathcal{P}_{N-q}\left(\mathcal{W}^{(0 \rightarrow N-1)}\right)-(-1)^{N-q} n_{2 q}}{n_{2 N-1}} .
$$

The slope parameters are then determined by inverting the matrix $X_{N-1, N-1}$

$$
B_{N-1}=X_{N-1, N-1}^{-1} A_{N-1} .
$$

By solving the above matrices, the slope parameters can be written as

$$
\begin{aligned}
& b_{1}=\frac{n_{1} n_{3}^{2} \omega_{1}}{n_{1} n_{2} n_{3}-n_{0} n_{3}^{2}-n_{1}^{2}} \text { for } N=2 \\
& b_{k}=\frac{\omega_{k} \prod_{j=1, j \neq k}^{N-1}\left(\omega_{k}^{2}-\omega_{j}^{2}\right)}{\sum_{j=1}^{N-1} Y_{j} \omega_{k}^{2 j-2}} \text { for } N \geqslant 3
\end{aligned}
$$

where $k \in[1 ; N-1]$ and

$$
Y_{j}=\frac{n_{2 j}-\frac{n_{0} n_{2 j+1}}{n_{1}}-\frac{n_{2 j-1}}{n_{2 N-1}}}{n_{2 N-1}} .
$$

One should note that this synthesis technique leads to a unique solution for a given specifications. So, there is theoretically only one transmission zero between two consecutive bands whatever the order of the designed filter.

\section{ImPlemENTATION With $L C$ ElEmENTS}

Starting from a classical ladder structure composed of series inductances and shunt capacitances, a low-pass prototype filter can be modified to use only parallel capacitances separated by $J$ admittance inverters [32]. Using the generalized frequency transformation technique presented in Section II, the $i^{\text {th }}$ capacitance, $C_{i}^{L P}$, of such a low-pass prototype can be transformed into a multiband bandpass resonator as shown in Fig. 2. The multiband bandpass resonator then obtained consists in the parallel association of one bandpass resonator $\left(L_{0}^{(i)} ; C_{0}^{(i)}\right)$ and $N-1$ band-stop ones $\left(L_{k}^{(i)} ; C_{k}^{(i)}\right), k \in[1 ; N-1]$ whose values are given by the following equations:

$$
\begin{aligned}
L_{0}^{(i)} & =\frac{1}{b_{0} C_{i}^{L P} \omega_{0}} \\
\text { ACCEPTED } &
\end{aligned}
$$

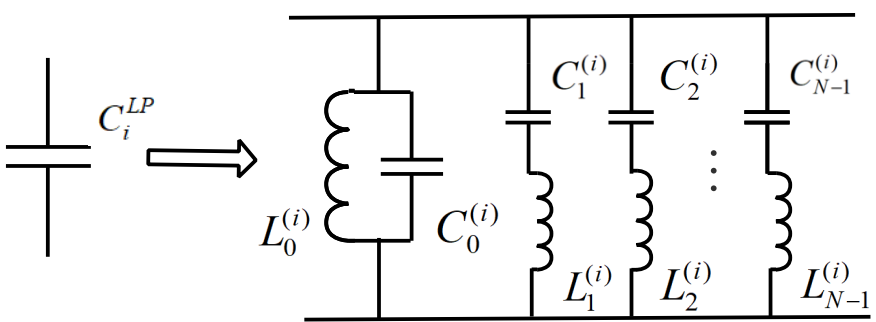

Fig. 2. Transformation of a low-pass prototype capacitance into a multiband bandpass resonator (MBPR).

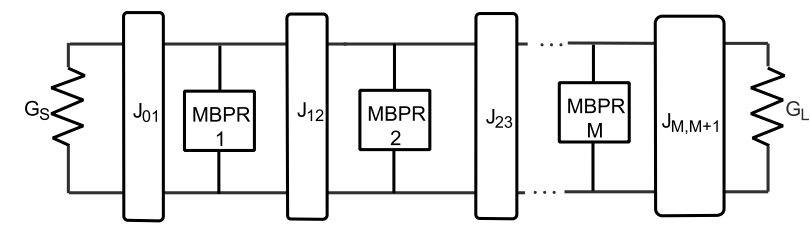

Fig. 3. Schematic representation of the proposed MBPF.

$$
\begin{aligned}
& C_{0}^{(i)}=\frac{1}{L_{0}^{(i)} \omega_{0}^{2}} \\
& L_{k}^{(i)}=\frac{b_{k}}{C_{i}^{L P} \omega_{k}} \\
& C_{k}^{(i)}=\frac{1}{L_{k}^{(i)} \omega_{k}^{2}} .
\end{aligned}
$$

A $M$-order MBPF is then composed of $M$ MBPRs separated by $J$ admittance inverters as shown in Fig. 3. The $J_{i, i+1}$ inverters are here defined in a classical way taking into account only the bandpass part of each MBPR [32]

$$
\begin{aligned}
J_{01} & =\sqrt{\frac{G_{S} \beta_{0}^{(1)}}{b_{0} g_{0} g_{1}}} \\
J_{i, i+1} & =\frac{1}{b_{0}} \sqrt{\frac{\beta_{0}^{(i)} \beta_{0}^{(i+1)}}{g_{i} g_{i+1}}} \\
J_{M, M+1} & =\sqrt{\frac{G_{L} \beta_{0}^{(M)}}{b_{0} g_{M} g_{M+1}}}
\end{aligned}
$$

where the $g_{i}$ coefficients are the low-pass prototype parameters, $G_{S}$ and $G_{L}$ are the source impedance, and $\beta_{0}^{(i)}=\omega_{0} C_{0}^{(i)}$.

To use only bandpass resonators, all the stopband $L C$-series elements can be transformed in $L C$-parallel ones using another set of J-inverters. A MBPR then consists only in LC-parallel resonators (see Fig. 4), the main one $\left(L_{0}^{(i)}, C_{0}^{(i)}\right)$ being separated from the others $\left(L_{k}^{(i)}, C_{k}^{(i)}\right), k \in[1 ; N-1]$, and $i \in[1 ; M]$, by new J-inverters defined as follows:

$$
J_{k}^{(i)}=\sqrt{\frac{\beta_{0}^{(i)} \beta_{k}^{(i)}}{b_{0} b_{k}}}
$$

with $\beta_{k}^{(i)}=\omega_{k} C_{k}^{(i)}$.

We should mention here that the values of capacitances both for bandpass and band-stop resonators can be chosen arbitrarily. However, in this article, considering the technological constraints, we have chosen the optimal capacitances 


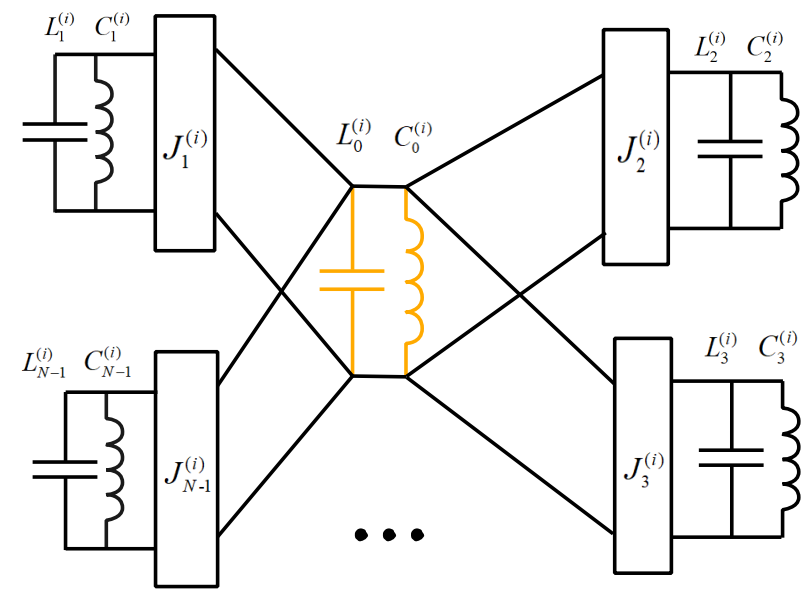

Fig. 4. MBPRs with parallel $L C$ resonators.

values for band-stop and bandpass resonators with a view to the implementation in microstrip technology.

\section{SyNTHESIS AND EXPERIMENTAL VALIDATION OF THREE TEST MBPFS}

For validating the frequency transformation method and the implementation proposed in the previous sections, three examples of MBPFs have been synthesized and experimentally validated with 3,4 , and 5 bands, respectively. In the first case (tri-band filter), all the output parameter expressions obtained from (12) to (21) are presented directly here as an example. In the other two cases, for the sake of clarity, these expressions are presented in Appendix (see Sections B and C).

The three test filters are of order 3 with a Chebyshev approximation and 20-dB bandpass return loss (RL). There are implemented in a low-cost microstrip technology using a RO4003C Rogers substrate (dielectric constant: $\varepsilon_{r}=3.55$, height: $h=0.508 \mathrm{~mm}$, dissipation factor: $\tan \delta=0.0027$ ) with copper metallization (metal thickness: $t=17.5 \mu \mathrm{m}$, conductivity: $\left.\sigma=5.8 \times 10^{7} \mathrm{~S} . \mathrm{m}^{-1}\right)$. All MBPRs use a star-like structure according to Fig. 4 topology connecting $N$ stubs at the same point allowing the implementation of such multiband filters without a significant increase of the circuit size compared to a single-band one. All prototypes were simulated and optimized using ADS software from Keysight Technologies $^{\odot}$. The fabrication of the circuits was made by laser engraving using a LPKF Protolaser U4.

\section{A. Triple-Band Bandpass Filters}

To synthesize a triple-band bandpass filter using the frequency transformation method proposed here, one needs to take $N=3$ in (12) to (21). The resonance angular frequencies and slope parameters are then given by

$$
\begin{aligned}
\omega_{0} & =\sqrt{-\frac{n_{0} n_{5}}{n_{1}}} \\
\omega_{1} & =\sqrt{\frac{-n_{3}-\sqrt{n_{3}^{2}-4 n_{5} n_{1}}}{2 n_{5}}}
\end{aligned}
$$

TABLE I

\begin{tabular}{|c|c|c|c|}
\hline \multicolumn{4}{|c|}{ Resonators $(i \in[1 ; 3])$} \\
\hline$L_{0}^{(i)}=1.567 \mathrm{nH}$ & \multicolumn{2}{|c|}{$L_{1}^{(i)}=1.689 \mathrm{nH}$} & $L_{2}^{(i)}=1.380 \mathrm{nH}$ \\
\hline$C_{0}^{(i)}=2.384 \mathrm{pF}$ & \multicolumn{2}{|c|}{$C_{1}^{(i)}=3.124 \mathrm{pF}$} & $C_{2}^{(i)}=2.321 \mathrm{pF}$ \\
\hline \multicolumn{2}{|c|}{$J_{1}^{(i)}=0.0072 \mathrm{~S}$} & \multicolumn{2}{|c|}{$J_{2}^{(i)}=0.0071 \mathrm{~S}$} \\
\hline \multicolumn{4}{|c|}{ Inverters $\left(J_{i, i+1}\right)$} \\
\hline \multicolumn{2}{|c|}{$J_{01}=J_{34}=0.012 \mathrm{~S}$} & \multicolumn{2}{|c|}{$J_{12}=J_{23}=0.0067 \mathrm{~S}$} \\
\hline
\end{tabular}

COMPONENT VAlues of THE IDEAL $L C$ TRI-BAND THIRD-ORDER BANDPASS FILTER

$$
\omega_{2}=\sqrt{\frac{-n_{3}+\sqrt{n_{3}^{2}-4 n_{5} n_{1}}}{2 n_{5}}}
$$

and

$$
\begin{aligned}
& b_{0}=\sqrt{\frac{-n_{0}}{n_{1} n_{5}}} \\
& b_{1}=\frac{\omega_{1}\left(\omega_{1}^{2}-\omega_{2}^{2}\right)}{Y_{1}+Y_{2} \omega_{1}^{2}} \\
& b_{2}=\frac{\omega_{2}\left(\omega_{2}^{2}-\omega_{1}^{2}\right)}{Y_{1}+Y_{2} \omega_{2}^{2}}
\end{aligned}
$$

with

$$
\begin{array}{r}
Y_{1}=\frac{n_{2}-\frac{n_{0} n_{3}}{n_{1}}-\frac{n_{1}}{n_{5}}}{n_{5}} \\
Y_{2}=\frac{n_{4}-\frac{n_{0} n_{5}}{n_{1}}-\frac{n_{3}}{n_{5}}}{n_{5}}
\end{array}
$$

where the $n_{p}(p \in[0 ; 5])$ are determined using (8) according to the specifications of the designed triple-band bandpass filter given in terms of low and high cut-off angular frequencies. The latter are then transposed in terms of resonant angular frequencies and slope parameters. As an example, the arbitrary following specifications are proposed.

1) Bandpass 1: 2.00-2.10 GHz (BW: $100 \mathrm{MHz}$ ).

2) Bandpass 2: 2.45-2.65 GHz (BW: $200 \mathrm{MHz}$ ).

3) Bandpass 3: 2.95-3.20 GHz (BW: $250 \mathrm{MHz}$ ).

The resulting resonant frequencies and slope parameters are $f_{0}=\omega_{0} / 2 \pi=2.604 \mathrm{GHz}, f_{1}=\omega_{1} / 2 \pi=2.191 \mathrm{GHz}$, $f_{2}=\omega_{2} / 2 \pi=2.812 \mathrm{GHz}, b_{0}=4.735, b_{1}=4.277$, and $b_{2}=5.818$. A tri-band third-order bandpass filter is first implemented in an ideal $L C$ structure (see Fig. 3), each resonator following the model of Fig. 4. All the resonator components and J-inverters, calculated from (25) to (32), are summarized in Table I. Note that the three tri-band resonators are identical in order to simplify the microstrip implementation in the next step. This is possible by fixing the three $C_{i}^{L P}$ components at the same value using an admittance scale factor when introducing the J-inverters in the low-pass prototype [32]. Fig. 5 shows the frequency response of this filter. The three simulated bands are in perfect agreement with the specifications.

The filter was then implemented in microstrip technology with the substrate characteristics given in the introduction of this section. Fig. 6 presents the layout of the fabricated 


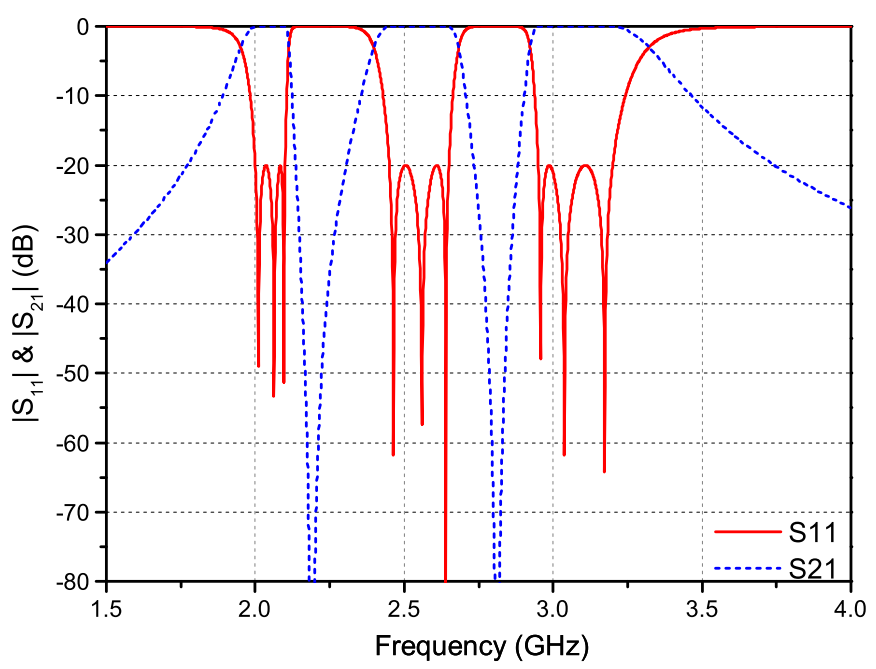

Fig. 5. Ideal frequency response of the specified triple-band bandpass filter.

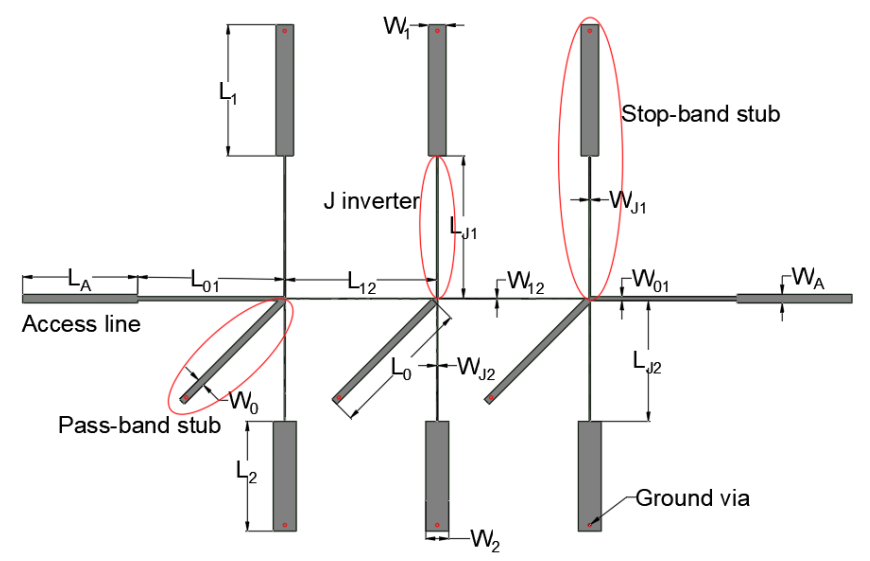

Fig. 6. Layout of the fabricated tri-band bandpass filter.

tri-band bandpass filter and Fig. 7 a picture of the fabricated filter together with the quad- and quint-band ones. It consists in three-branched stars, allowing to keep the filter compact. Each star is made of a tri-band resonator together with its previous and next inverters. A tri-band resonator consists in one bandpass element, i.e., a short-circuit stub of length $\lambda / 4$ at $\omega_{0}$, and two stopband ones consisting of a short-circuit stub of length $\lambda / 4$ at $\omega_{1}$ and $\omega_{2}$, respectively, and separated from the connection point by another quarter-wavelength line at the same respective frequency having the J-inverter role. Compared with the method described in [30], our proposal allows a direct determination of the values of circuit elements from the transformation formulas. For implementation, as mentioned in Section III, we have chosen suitable values to avoid too narrow (less than $0.1 \mathrm{~mm}$ ) or too wide (more than $4 \mathrm{~mm}$ ) linewidth. All lengths and widths of the resonators and the inverters are summarized in Table II. The tri-band filter is $72.1 \times 68.5 \mathrm{~mm}^{2}$ without taking account for the two $50 \Omega$ access lines.

Fig. 8 presents the EM-simulated and measured frequency responses of the fabricated tri-band bandpass filter. The correlation between both responses is very good over the entire frequency band. The measured RL are 12.3, 14.9, and $14.1 \mathrm{~dB}$ from the first to the last bandpass and the measured

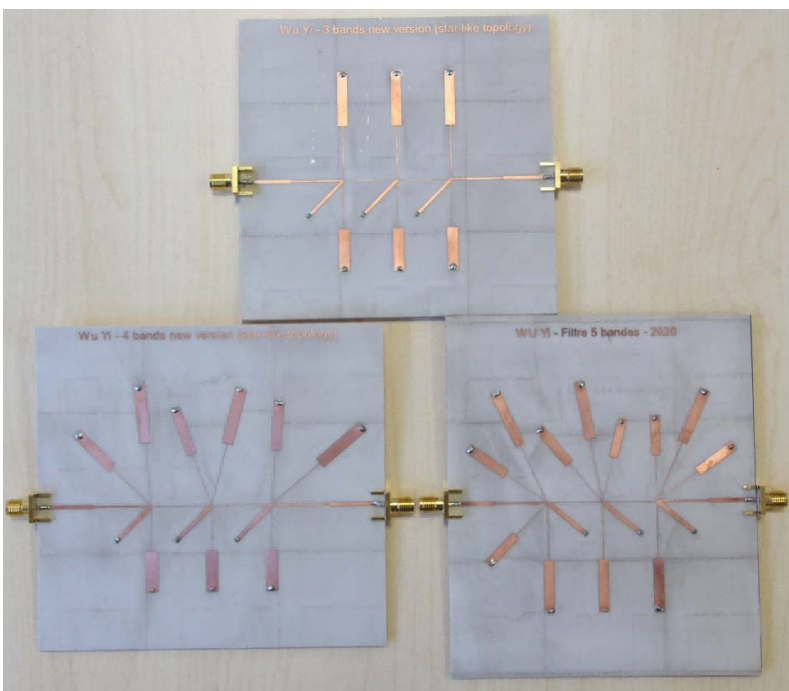

Fig. 7. Photograph of the three fabricated filters: tri-band (top), quad-band (bottom left), and quint-band (bottom right).

TABLE II

Microstrip TRI-BAND THIRD-ORdER BANDPASS FILTER: LENGTH AND WIDTH DiMENSIONS (IN MM)

\begin{tabular}{|l|l|c|}
\hline \multicolumn{3}{|c|}{ Resonators } \\
\hline$L_{0}=17.50$ & $L_{1}=18.80$ & $L_{2}=14.50$ \\
\hline$W_{0}=0.92$ & $W_{1}=3.36$ & $W_{2}=3.50$ \\
\hline$L_{J 1}=18.70$ & & $L_{J 2}=16.50$ \\
\hline$W_{J 1}=0.15$ & & $W_{J 2}=0.12$ \\
\hline \multicolumn{3}{|c|}{ Inverters } \\
\hline$L_{01}=17.70$ & & $L_{12}=18.34$ \\
\hline$W_{01}=0.51$ & & $W_{12}=0.11$ \\
\hline
\end{tabular}
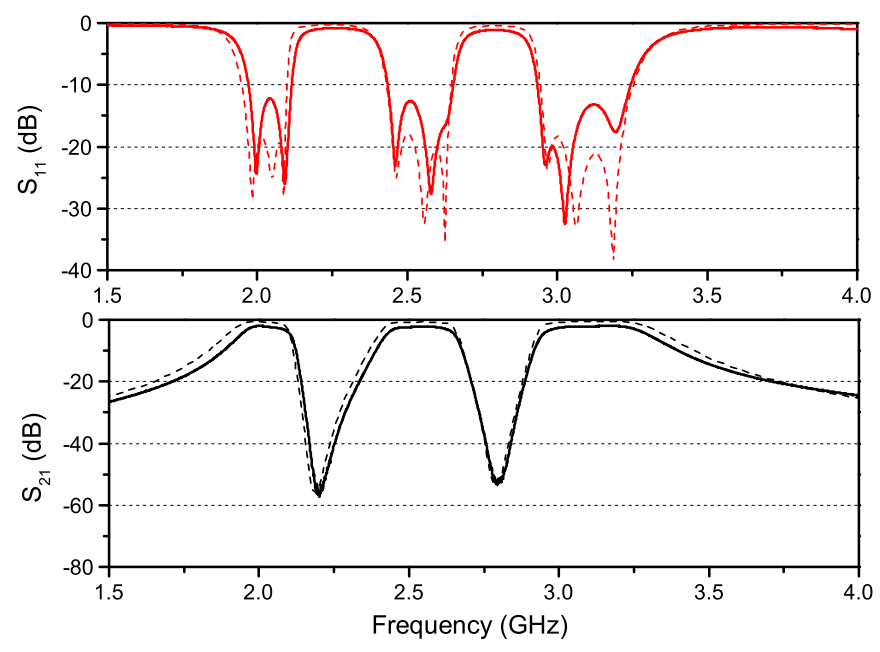

Fig. 8. EM-simulated (dotted lines) and measured (solid lines) S-parameters of the triple-band bandpass filter.

insertion loss (IL) are 1.98, 2.17, and $2.01 \mathrm{~dB}$, respectively. The isolation between the three bandpass are better than 58.1 and $57.6 \mathrm{~dB}$.

\section{B. Quad-Band Bandpass Filters}

In the same way as for the tri-band case, the synthesis of a quad-band bandpass filter using this frequency transformation 
TABLE III

Component VALUeS of THE IDEAL LC QUAD-BAND THIRD-ORDER BANDPASS FILTER

\begin{tabular}{|c|c|c|}
\hline \multicolumn{3}{|c|}{ Resonators $(i \in[1 ; 3])$} \\
\hline$L_{0}^{(i)}=1.586 \mathrm{nH}$ & $L_{1}^{(i)}=1.902 \mathrm{nH}$ & $L_{2}^{(i)}=1.603 \mathrm{nH}$ \\
\hline$C_{0}^{(i)}=2.412 \mathrm{pF}$ & $C_{1}^{(i)}=2.892 \mathrm{pF}$ & $C_{2}^{(i)}=2.355 \mathrm{pF}$ \\
\hline \multicolumn{2}{|c|}{$L_{3}^{(i)}=1.315 \mathrm{nH}$} & $C_{3}^{(i)}=2.211 \mathrm{pF}$ \\
\hline$J_{1}^{(i)}=0.0078 \mathrm{~S}$ & $J_{2}^{(i)}=0.0074 \mathrm{~S}$ & $J_{3}^{(i)}=0.0067 \mathrm{~S}$ \\
\hline \multicolumn{3}{|c|}{ Inverters $\left(J_{i, i+1}\right)$} \\
\hline$J_{01}=J_{34}=0$ & $J_{12}$ & $23=0.0074 \mathrm{~S}$ \\
\hline
\end{tabular}

method starts with the determination of the expression of the resonant angular frequencies and the associated slope parameters as functions of the low and high cut-off frequencies. To do so, one takes $N=4$ in (12) to (21). The resulting equations are given in Appendix B for a sake of clarity.

Arbitrary specifications are also proposed here as an example to design, fabricate, and measure a third-order quad-band bandpass filter. Note that a first example of quad-band bandpass filter was already presented in [33] with four equal absolute bandwidths. The equations presented in [33] and here are slightly different in their writing (especially the $n_{p}$ coefficients) but completely equivalent. This is only because the generalization to any number of bands was not established at the time [33] was written. The specifications proposed in this article show different bandwidths for each band are as follows:

1) Bandpass 1: 2.00-2.08 GHz (BW: $80 \mathrm{MHz}$ ).

2) Bandpass 2: $2.30-2.40 \mathrm{GHz}(\mathrm{BW}: 100 \mathrm{MHz}$ ).

3) Bandpass 3: $2.65-2.80 \mathrm{GHz}(\mathrm{BW}: 150 \mathrm{MHz})$.

4) Bandpass 4: $3.04-3.20 \mathrm{GHz}(\mathrm{BW}: 160 \mathrm{MHz}$ ).

The resulting resonant frequencies and slope parameters are $f_{0}=\omega_{0} / 2 \pi=2.573 \mathrm{GHz}, f_{1}=\omega_{1} / 2 \pi=2.146 \mathrm{GHz}$, $f_{2}=\omega_{2} / 2 \pi=2.501 \mathrm{GHz}, f_{3}=\omega_{3} / 2 \pi=2.951 \mathrm{GHz}, b_{0}=$ 5.251, $b_{1}=6.381, b_{2}=5.697$, and $b_{3}=8.066$. An ideal $L C$ structure implementation is then made as in the tri-band case and all the resonators are again fixed identical by taking all the $C_{i}^{L P}$ equal. All the resonator component values and J-inverter ones are summarized in Table III. As shown in Fig. 9, the four obtained bands are, as previously, in perfect agreement with the specifications.

Fig. 10 presents the layout of the fabricated quad-band bandpass filter and a picture is also shown in Fig. 7. Again, three stars separated by J-inverters are obtained, each star including six branches this time: one bandpass element, three stopband ones, and the previous and next J-inverters. All lengths and widths of the resonators and the inverters are summarized in Table IV. The quad-band filter is $97.7 \times 68.9 \mathrm{~mm}^{2}$ without taking into account the two $50 \Omega$ access lines.

Fig. 11 presents the EM-simulated and measured frequency responses of the fabricated quad-band bandpass filter. As in the tri-band case, both responses are in near perfect agreement. The measured RL are 12.1, 14.2, 14.2, and $14.3 \mathrm{~dB}$ from the first to the fourth bandpass and the measured IL are 2.90, 2.94, 2.95 , and $2.65 \mathrm{~dB}$, respectively. Very good isolation are here again achieved between all the bandpass.

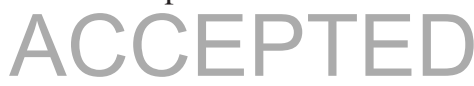

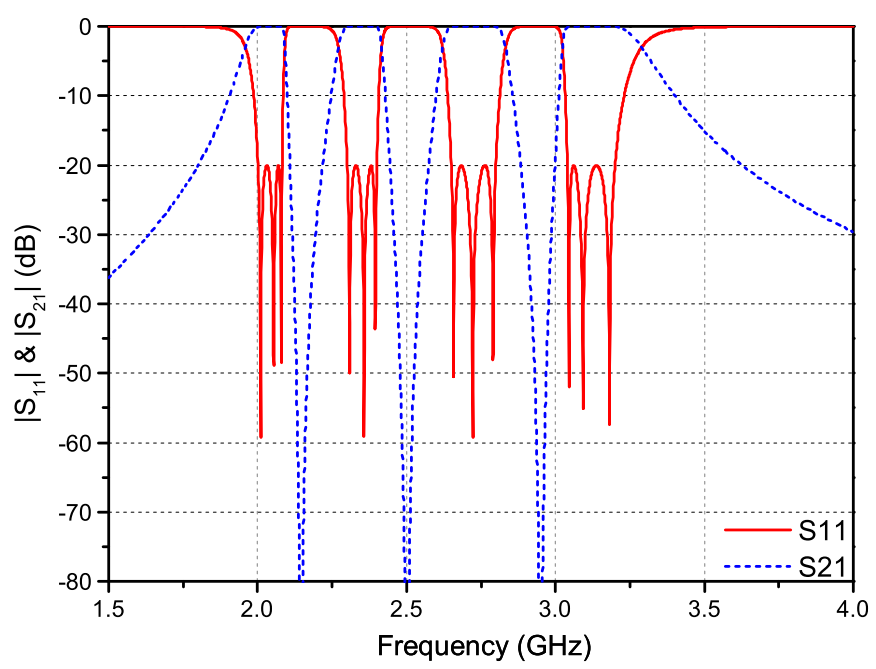

Fig. 9. Ideal frequency response of the specified quad-band bandpass filter.

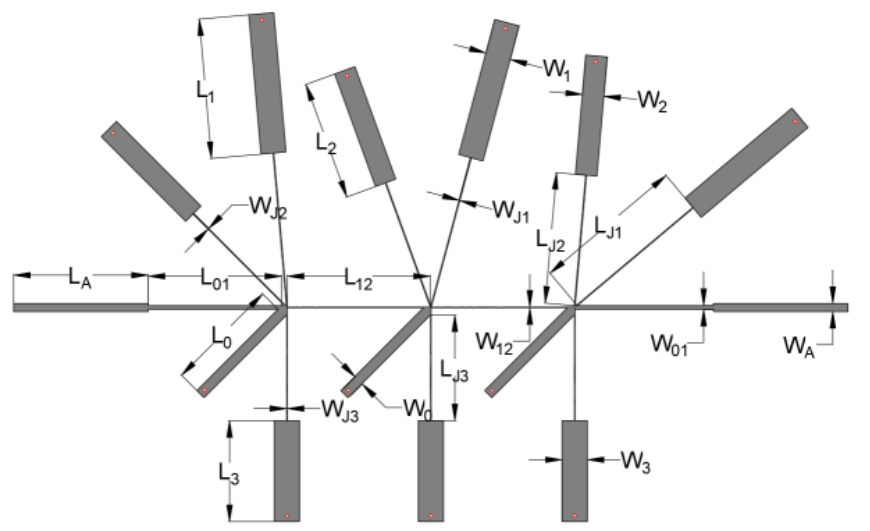

Fig. 10. Layout of the fabricated quad-band bandpass filter.

TABLE IV

MICROSTRIP QUAD-BAND THIRD-ORDER BANDPASS FILTER: LENGTH AND WIDTH DIMENSIONS (IN MM)

\begin{tabular}{|c|c|c|c|}
\hline \multicolumn{4}{|c|}{ Resonators } \\
\hline$L_{0}=16.70$ & $L_{1}=19.01$ & $L_{2}=16.2$ & $L_{3}=15.30$ \\
\hline$W_{0}=1.30$ & $W_{1}=3.80$ & $W_{2}=3.30$ & $W_{3}=3.51$ \\
\hline$L_{J 1}=21.30$ & $L_{J 2}=18.40$ & $L_{J 3}=15.70$ \\
\hline$W_{J 1}=0.11$ & $W_{J 2}=0.12$ & $W_{J 3}=0.10$ \\
\hline \multicolumn{3}{|c|}{ Inverters } \\
\hline \multicolumn{2}{|c|}{$L_{01}=19.23$} & $L_{12}=19.85$ \\
\hline$W_{01}=0.51$ & & $W_{12}=0.11$ \\
\hline
\end{tabular}

\section{Quint-Band Bandpass Filters}

In the same way as for the two previous cases, one can derive the equations of the resonant angular frequencies and the slope parameters for the quint-band bandpass filters as functions of the low and high cut-off angular frequencies by taking $N=5$ in (12) to (21). The resulting equations are given in Appendix $\mathrm{C}$ for the sake of clarity.

The arbitrary specifications proposed here to design an example of quint-band bandpass filter are as follows: 


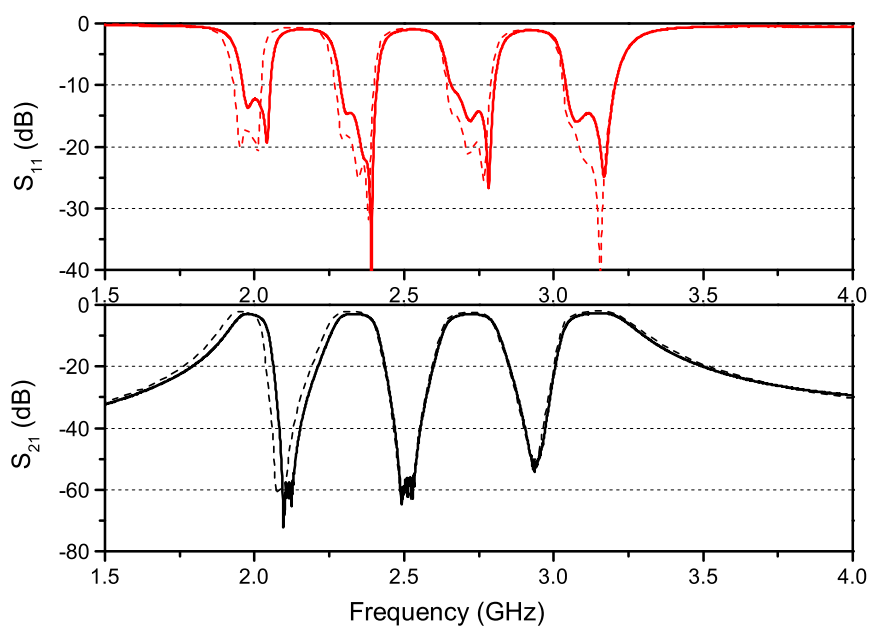

Fig. 11. EM-simulated (dotted lines) and measured (solid lines) S-parameters of the quad-band bandpass filter.

TABLE V

Component Values of THE Ideal LC Quint-Band THIRD-ORDER BANDPASS FILTER

\begin{tabular}{|c|c|c|c|}
\hline \multicolumn{4}{|c|}{ Resonators $(i \in[1 ; 3])$} \\
\hline$L_{0}^{(i)}=1.602 \mathrm{nH}$ & \multicolumn{2}{|c|}{$L_{1}^{(i)}=1.874 \mathrm{nH}$} & $L_{2}^{(i)}=1.645 \mathrm{nH}$ \\
\hline \multicolumn{2}{|c|}{$L_{3}^{(i)}=1.388 \mathrm{nH}$} & \multicolumn{2}{|c|}{$L_{4}^{(i)}=1.241 \mathrm{nH}$} \\
\hline$C_{0}^{(i)}=2.436 \mathrm{pF}$ & \multicolumn{2}{|c|}{$C_{1}^{(i)}=2.850 \mathrm{pF}$} & $C_{2}^{(i)}=2.502 \mathrm{pF}$ \\
\hline \multicolumn{2}{|c|}{$C_{3}^{(i)}=2.333 \mathrm{pF}$} & \multicolumn{2}{|c|}{$C_{4}^{(i)}=2.080 \mathrm{pF}$} \\
\hline \multicolumn{2}{|c|}{$J_{1}^{(i)}=0.0070 \mathrm{~S}$} & \multicolumn{2}{|c|}{$J_{2}^{(i)}=0.0072 \mathrm{~S}$} \\
\hline \multicolumn{2}{|c|}{$J_{3}^{(i)}=0.0069 \mathrm{~S}$} & \multicolumn{2}{|c|}{$J_{4}^{(i)}=0.0064 S$} \\
\hline \multicolumn{4}{|c|}{ Inverters $\left(J_{i, i+1}\right)$} \\
\hline \multicolumn{2}{|c|}{$J_{01}=J_{34}=0.013 \mathrm{~S}$} & \multicolumn{2}{|c|}{$J_{12}=J_{23}=0.0069 \mathrm{~S}$} \\
\hline
\end{tabular}

1) Bandpass 1: 2.00-2.10 GHz (BW: $100 \mathrm{MHz}$ ).

2) Bandpass 2: $2.30-2.38 \mathrm{GHz}(\mathrm{BW}: 80 \mathrm{MHz})$.

3) Bandpass 3: 2.60-2.69 GHz (BW: $90 \mathrm{MHz}$ ).

4) Bandpass 4: 2.88-2.98 GHz (BW: $100 \mathrm{MHz}$ ).

5) Bandpass 5: 3.20-3.30 GHz (BW: $100 \mathrm{MHz}$ ).

They leads to the following resonant frequencies and slope parameters: $f_{0}=\omega_{0} / 2 \pi=2.548 \mathrm{GHz}, f_{1}=\omega_{1} / 2 \pi=$ $2.178 \mathrm{GHz}, f_{2}=\omega_{2} / 2 \pi=2.480 \mathrm{GHz}, f_{3}=\omega_{3} / 2 \pi=$ $2.797 \mathrm{GHz}, f_{4}=\omega_{4} / 2 \pi=3.137 \mathrm{GHz}, b_{0}=5.421$, $b_{1}=6.075, b_{2}=5.735, b_{3}=8.323$, and $b_{4}=10.050$. An ideal $L C$ quint-band bandpass filter is also implemented to validate the method. The resonator component and J-inverter values are summarized in Table $\mathrm{V}$ and the frequency responses shown in Fig. 12. Once again, all the bands are in perfect agreement with the specifications.

Fig. 13 presents the layout of the fabricated quint-band bandpass filter and a picture is also shown in Fig. 7. Three stars separated by J-inverters are obtained, each star including seven branches with an additional stopband one compared to the quad-band case. All lengths and widths of the resonators and the inverters are summarized in Table VI. The quint-band filter is $91.5 \times 77.3 \mathrm{~mm}^{2}$ without taking into account the two $50 \Omega$ access lines.

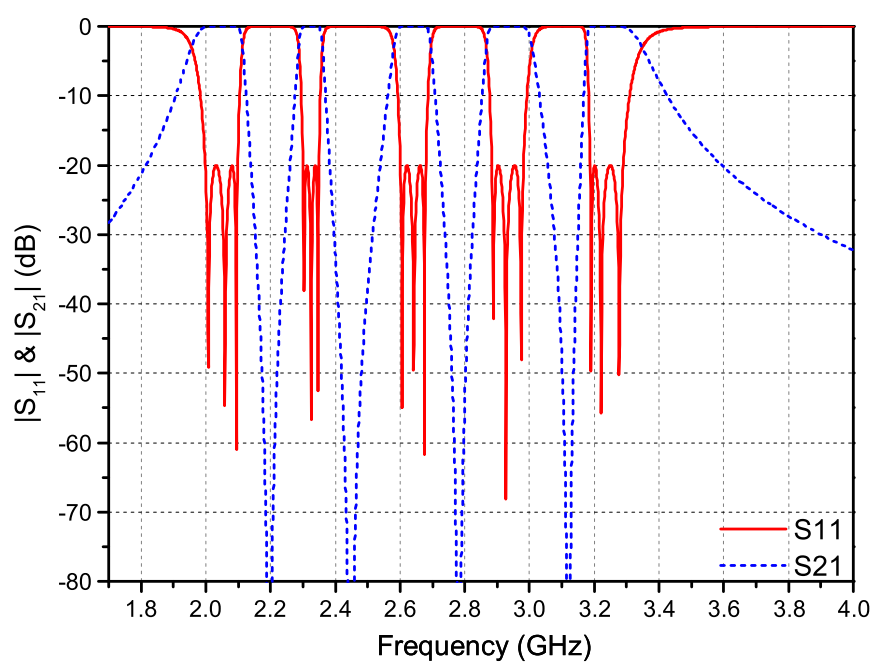

Fig. 12. Ideal frequency response of the specified quint-band bandpass filter.

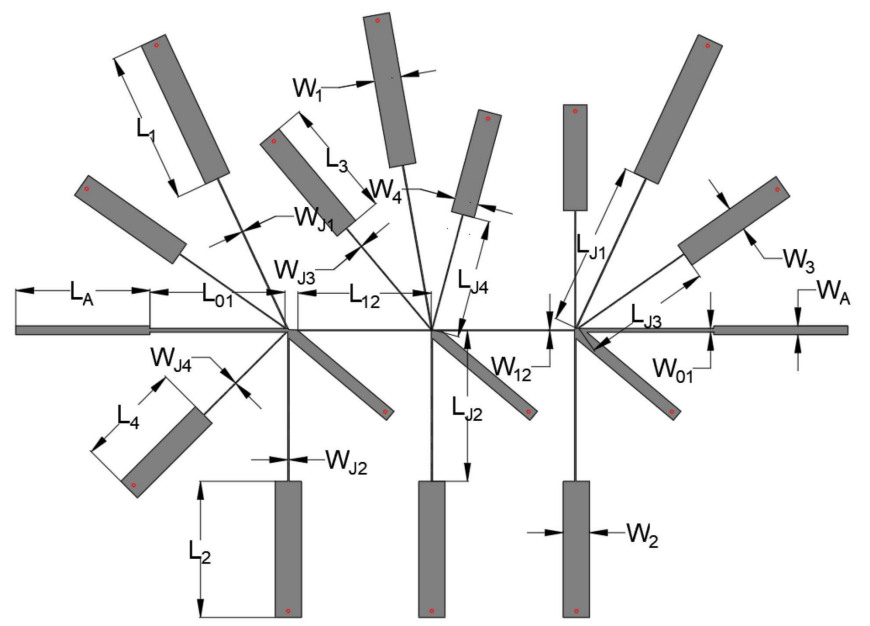

Fig. 13. Layout of the fabricated quint-band bandpass filter.

TABLE VI

Microstrip QUINT-BAND THIRD-ORdER BANDPASS FILTER: LENGTH AND WIDTH DiMENSIONS (IN MM)

\begin{tabular}{|c|c|c|c|c|}
\hline \multicolumn{5}{|c|}{ Resonators } \\
\hline$L_{0}=17.73$ & \multicolumn{2}{|c|}{$L_{1}=19.50$} & \multicolumn{2}{|c|}{$L_{2}=17.40$} \\
\hline$W_{0}=1.55$ & \multicolumn{2}{|c|}{$W_{1}=3.44$} & \multicolumn{2}{|c|}{$W_{2}=3.36$} \\
\hline \multicolumn{2}{|c|}{$L_{3}=15.33$} & \multicolumn{3}{|c|}{$L_{4}=13.50$} \\
\hline \multicolumn{2}{|c|}{$W_{3}=3.10$} & \multicolumn{3}{|c|}{$W_{4}=3.01$} \\
\hline$L_{J 1}=21.40$ & $L_{J 2}=19.30$ & \multicolumn{2}{|c|}{$L_{J 3}=17.05$} & $L_{J 4}=15.33$ \\
\hline$W_{J 1}=0.14$ & $W_{J 2}=0.17$ & \multicolumn{2}{|c|}{$W_{J 3}=0.10$} & $W_{J 4}=0.10$ \\
\hline \multicolumn{5}{|c|}{ Inverters } \\
\hline \multicolumn{2}{|c|}{$L_{01}=17.71$} & \multicolumn{3}{|c|}{$L_{12}=18.34$} \\
\hline \multicolumn{2}{|c|}{$W_{01}=0.50$} & \multicolumn{3}{|c|}{$W_{12}=0.11$} \\
\hline
\end{tabular}

Fig. 14 presents the simulated and measured frequency responses of the fabricated quint-band bandpass filter. Here again, simulations and measurements are in a good correlation, except a slight frequency shift in the higher transmission zeros. This can be explained by the low-cost technology process, which implies in particular that the vias were made by hand. 


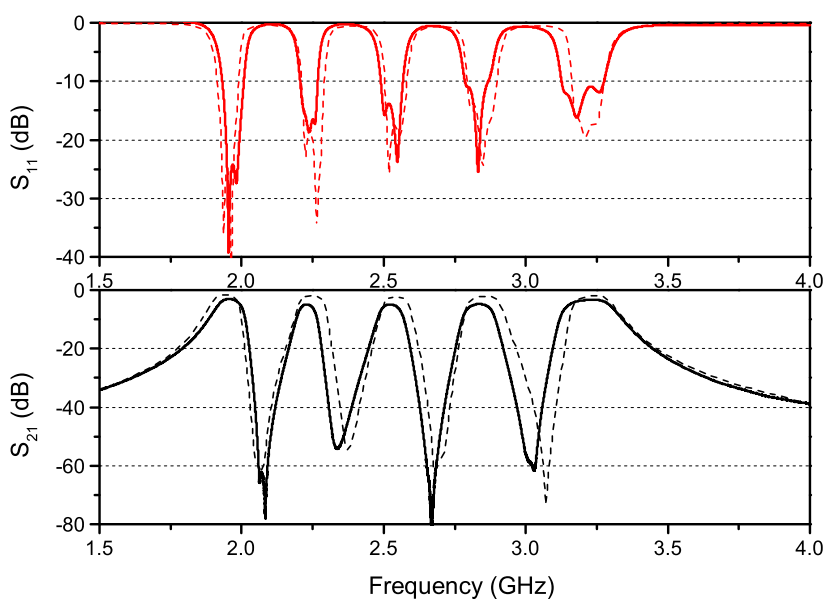

Fig. 14. EM-simulated (dotted lines) and measured (solid lines) S-parameters of the quint-band bandpass filter.

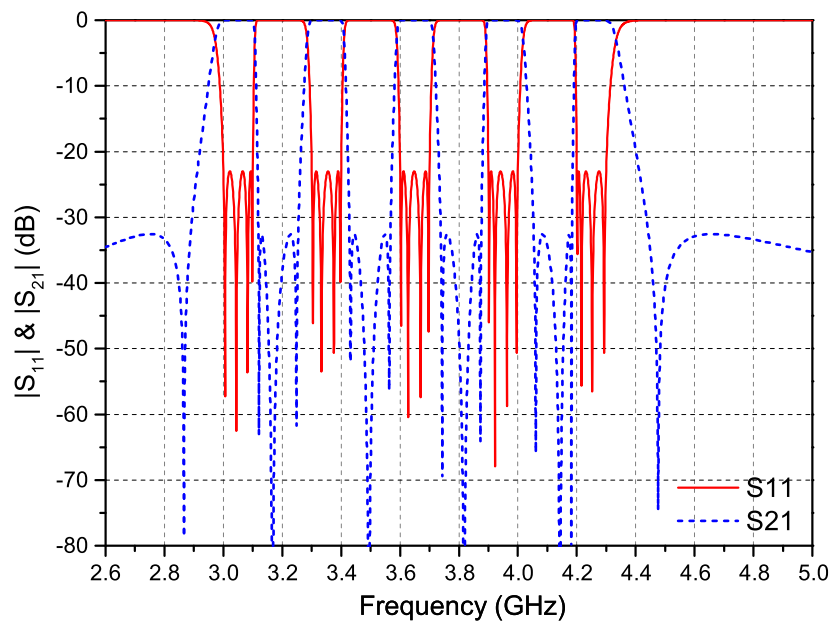

Fig. 15. Ideal frequency response of the specified quint-band elliptic bandpass filter.

The measured RL are $24.8,17.5,13.9,10.9$, and $10.8 \mathrm{~dB}$ from the first to the fifth band and the measured IL are 3.01, 4.95, $4.98,4.75$, and $3.45 \mathrm{~dB}$, respectively. Very good isolation are again achieved between all the bandpass.

\section{DISCUSSION}

Using our proposed transform function, we always assume that lower cut-off angular frequencies $\omega_{L_{i}}$ and the upper cut-off angular frequencies $\omega_{H_{i}}(i \in[1 ; N])$ are mapped to -1 and +1 in the $\Omega$ normalized symmetrical domain, respectively. This features that only transfer functions that are symmetric in the low-pass domain can be used in this method. Chebyshev, Butterworth, and elliptic filters have all symmetrical structures where the symmetry is defined as $g_{0}=g_{n+1}, g_{1}=g_{n}, g_{2}=g_{n-1}$, etc., in the low-pass domain. There are all therefore compatible with our proposed synthesis method. For Gaussian low-pass prototype (flat-group-delay), filters with order $n=1$ are identical to the first-order Butterworth low-pass prototype and can also be synthesized with this method. However, Gaussian filters with order $n \geq 2$ are structurally asymmetrical as discussed in [22]. It is therefore impossible to synthesize multiband filter with this method. Nevertheless, flat group-delay can also be obtained with symmetrical structures using cross-coupling as presented in [34] and [35].

As an example, we present a multiband elliptic filter with quint-band response. We consider a fourth-order filter with an elliptic approximation (23-dB RL with normalized TZs at $\pm 2.2 j$ ). The specifications for this case are as follows:

1) Bandpass 1: 3.00-3.10 GHz (BW: $100 \mathrm{MHz})$.

2) Bandpass 2: 3.30-3.40 GHz (BW: $100 \mathrm{MHz})$.

3) Bandpass 3: 3.60-3.70 GHz (BW: $100 \mathrm{MHz})$.

4) Bandpass 4: 3.90-4.00 GHz (BW: $100 \mathrm{MHz})$.

5) Bandpass 5: 4.20-4.30 GHz (BW: $100 \mathrm{MHz}$ ).

The resonating frequencies and slope parameters can be easily obtained by the generalized formulas in Section II. Each sub-band is a bandwidth-scaled and frequency-translated copy of the original low-pass prototype (i.e., each bandpass reaches 23-dB in-band RL and owns two TZs on both sides, as shown in Fig. 15). This example also shows that it is possible to introduce several TZs between each bandpass with this method using elliptic filters.

\section{CONCLUSION}

In this article, a new generalized synthesis method for designing MBPF has been proposed. This method is relatively simple and leads to a completely analytical equation system. It uses slope parameters offering flexibility to implement the designed filters with noncoupled structures, using them directly, or with coupled ones calculating the coupling coefficients from these parameters [36]-[38]. Note that from six bands and beyond a numerical resolution of (14) will be necessary because of the degree of this equation.

Three third-order MBPFs with a different number of bands (3, 4, and 5, respectively) were implemented in microstrip technologies to illustrate the efficiency of the synthesis method. A star-like structure has been chosen to realize each multiband bandpass resonator of the filters. This enabled to keep the filters compact and the implementation simple. Very good agreement has been achieved between theoretical responses and measurements. This implementation solution is in fact mainly limited by the maximum bandwidth that can be obtained with the bandpass elements. Increasing the number of bands would lead to narrow bandwidth for each band and sharp rejection between them. Such a rejection could be difficult to realize because it implies that the impedance of J-inverters separating the bandpass part to the stopband ones in each multiband resonator will increase potentially reaching technological limits. Nevertheless, such an implementation is just an example, which has the interests to be low cost and easy to do, and any other topology or technology allowing wider bandwidths can be used.

\section{APPENDIX A}

\section{EXPRESSION GOVERNING THE RESONANT ANGULAR FREQUENCIES OF THE BAND-STOP RESONATORS}

As presented in Section II, the resonant angular frequencies of the stopband parts of an $N$-band bandpass resonator (MBPR) are the positive solutions of (14). 
In the case of an $N$-band bandpass filters, the coefficients $n_{p}$ of the $U^{(N)}(\omega)$ function [see (3)] are given by (10) in the case of an odd value of $p(p=2 q-1, q \in[1 ; N])$.

For $q=N$ and so $p=2 N-1$, this equation leads to

$$
n_{2 N-1}=-\frac{\omega_{0}}{b_{0}}
$$

knowing that $\mathcal{P}_{0}\left(\mathcal{W}^{(1 \rightarrow N-1)}\right)=1$ as defined in Section II-A. So, (10) becomes

$$
\begin{aligned}
n_{p} & =n_{2 q-1} \\
& =(-1)^{N-q} n_{2 N-1} \mathcal{P}_{N-q}\left(\mathcal{W}^{(1 \rightarrow N-1)}\right) .
\end{aligned}
$$

Applying (42) with $q=N-1(p=2 N-3)$ leads to

$$
n_{2 N-3}=-n_{2 N-1} \mathcal{P}_{1}\left(\mathcal{W}^{(1 \rightarrow N-1)}\right) .
$$

Considering any angular frequency of the stopband parts $\omega_{k}, k \in[1 ; N-1]$, this expression can be written as follows:

$$
n_{2 N-3}=-n_{2 N-1}\left[\mathcal{P}_{1}\left(\mathcal{W}_{\{k\}}^{(1 \rightarrow N-1)}\right)+\omega_{k}^{2}\right]
$$

which implies that

$$
n_{2 N-1} \mathcal{P}_{1}\left(\mathcal{W}_{\{k\}}^{(1 \rightarrow N-1)}\right)=-\left[n_{2 N-3}+n_{2 N-1} \omega_{k}^{2}\right] .
$$

In the same way, for $q=N-2(p=2 N-5)$, (42) becomes

$$
\begin{aligned}
n_{2 N-5} & =n_{2 N-1} \mathcal{P}_{2}\left(\mathcal{W}^{(1 \rightarrow N-1)}\right) \\
& =n_{2 N-1}\left[\mathcal{P}_{2}\left(\mathcal{W}_{\{k\}}^{(1 \rightarrow N-1)}\right)+\omega_{k}^{2} \mathcal{P}_{1}\left(\mathcal{W}_{\{k\}}^{(1 \rightarrow N-1)}\right)\right] \\
& =n_{2 N-1} \mathcal{P}_{2}\left(\mathcal{W}_{\{k\}}^{(1 \rightarrow N-1)}\right)-\left[n_{2 N-3} \omega_{k}^{2}+n_{2 N-1} \omega_{k}^{4}\right]
\end{aligned}
$$

which leads to

$$
n_{2 N-1} \mathcal{P}_{2}\left(\mathcal{W}_{\{k\}}^{(1 \rightarrow N-1)}\right)=n_{2 N-5}+n_{2 N-3} \omega_{k}^{2}+n_{2 N-1} \omega_{k}^{4} .
$$

Continuing thus for the other values of $q$ from $N-1$ to 1 , one can show that

$$
n_{2 N-1} \mathcal{P}_{N-q}\left(\mathcal{W}_{\{k\}}^{(1 \rightarrow N-1)}\right)=\sum_{r=0}^{N-q} n_{2(q+r)-1} \omega_{k}^{2 r} .
$$

Let's focus now on the case $q=1$ ( $p=1)$. First, using (42), we have

$$
\begin{aligned}
n_{1} & =(-1)^{N-1} n_{2 N-1} \mathcal{P}_{N-1}\left(\mathcal{W}^{(1 \rightarrow N-1)}\right) \\
& =(-1)^{N-1} \omega_{k}^{2} n_{2 N-1} \mathcal{P}_{N-2}\left(\mathcal{W}_{\{k\}}^{(1 \rightarrow N-1)}\right) .
\end{aligned}
$$

Using (48), this expression becomes

$$
\begin{aligned}
n_{1} & =(-1)^{N-1} \omega_{k}^{2} \frac{1}{(-1)^{N-2}} \sum_{r=0}^{N-2} n_{2 r+3} \omega_{k}^{2 r} \\
& =-\sum_{r=1}^{N-1} n_{2 r+1} \omega_{k}^{2 r} .
\end{aligned}
$$

Therefore, we obtain the equation governing the resonant angular frequencies of the band-stop resonators

$$
\sum_{r=0}^{N-1} n_{2 r+1} \omega_{k}^{2 r}=0 .
$$

This equation has $2(N-1)$ solutions, one half being positive and the other half negative. Of course, in this case, only the positive solutions are retained.

\section{APPENDIX B}

Resonant Angular Frequencies and Slope PARAMETERS FOR A QUAD-BAND BANDPASS FILTER

$$
\begin{aligned}
& \omega_{0}=\sqrt{\frac{-n_{0} n_{7}}{n_{1}}} \\
& \omega_{1}=\sqrt{-\frac{n_{5}}{3 n_{7}}+\sqrt[3]{\Omega_{1}}+\sqrt[3]{\Omega_{2}}} \\
& \omega_{2}=\sqrt{-\frac{n_{5}}{3 n_{7}}+\Delta_{3} \sqrt[3]{\Omega_{1}}+\Delta_{4} \sqrt[3]{\Omega_{2}}} \\
& \omega_{3}=\sqrt{-\frac{n_{5}}{3 n_{7}}+\Delta_{4} \sqrt[3]{\Omega_{1}}+\Delta_{3} \sqrt[3]{\Omega_{2}}} \\
& b_{0}=\sqrt{\frac{-n_{0}}{n_{1} n_{7}}} \\
& b_{1}=\frac{\omega_{1}\left(\omega_{1}^{2}-\omega_{2}^{2}\right)\left(\omega_{1}^{2}-\omega_{3}^{2}\right)}{Y_{3} \omega_{1}^{4}+Y_{2} \omega_{1}^{2}+Y_{1}} \\
& b_{2}=\frac{\omega_{2}\left(\omega_{2}^{2}-\omega_{1}^{2}\right)\left(\omega_{2}^{2}-\omega_{3}^{2}\right)}{Y_{3} \omega_{2}^{4}+Y_{2} \omega_{2}^{2}+Y_{1}} \\
& b_{3}=\frac{\omega_{3}\left(\omega_{3}^{2}-\omega_{1}^{2}\right)\left(\omega_{3}^{2}-\omega_{2}^{2}\right)}{Y_{3} \omega_{3}^{4}+Y_{2} \omega_{3}^{2}+Y_{1}}
\end{aligned}
$$

where

$$
\begin{aligned}
\Omega_{1} & =\Delta_{1}+\sqrt{\Delta_{1}^{2}+\Delta_{2}^{3}} \\
\Omega_{2} & =\Delta_{1}-\sqrt{\Delta_{1}^{2}+\Delta_{2}^{3}} \\
\Delta_{1} & =\frac{n_{5} n_{3}}{6 n_{7}^{2}}-\frac{n_{5}^{3}}{27 n_{7}^{3}}-\frac{n_{1}}{2 n_{7}} \\
\Delta_{2} & =\frac{n_{3}}{3 n_{7}}-\frac{n_{5}^{2}}{9 n_{7}^{2}} \\
\Delta_{3} & =\frac{-1+j \sqrt{3}}{2} \\
\Delta_{4} & =\frac{-1-j \sqrt{3}}{2} \\
Y_{1} & =\frac{n_{2}-\frac{n_{0} n_{3}}{n_{1}}-\frac{n_{1}}{n_{7}}}{n_{7}} \\
Y_{2} & =\frac{n_{4}-\frac{n_{0} n_{5}}{n_{1}}-\frac{n_{3}}{n_{7}}}{n_{7}} \\
Y_{3} & =\frac{n_{6}-\frac{n_{0} n_{7}}{n_{1}}-\frac{n_{5}}{n_{7}}}{n_{7}} .
\end{aligned}
$$

\section{APPENDIX C}

Resonant Angular Frequencies and Slope PARAMETERS FOR A QUAD-BAND BANDPASS FILTER

$$
\begin{aligned}
& \omega_{0}=\sqrt{-\frac{n_{0} n_{9}}{n_{1}}} \\
& \omega_{1}=\sqrt{-\frac{n_{7}}{4 n_{9}}-\frac{1}{2}\left(\sqrt{R_{0}}+\sqrt{R_{1}}\right)} \\
& \omega_{2}=\sqrt{-\frac{n_{7}}{4 n_{9}}-\frac{1}{2}\left(\sqrt{R_{0}}-\sqrt{R_{1}}\right)}
\end{aligned}
$$




$$
\begin{gathered}
\omega_{3}=\sqrt{-\frac{n_{7}}{4 n_{9}}+\frac{1}{2}\left(\sqrt{R_{0}}-\sqrt{R_{1}}\right)} \\
\omega_{4}=\sqrt{-\frac{n_{7}}{4 n_{9}}+\frac{1}{2}\left(\sqrt{R_{0}}+\sqrt{R_{1}}\right)}
\end{gathered}
$$

where

$$
\begin{aligned}
& R_{0}=\left(\frac{n_{7}}{2 n_{9}}\right)^{2}-\frac{2 n_{5}}{3 n_{9}}+R_{3} \\
& +\frac{n_{2}^{5}-3 n_{3} n_{7}+12 n_{1} n_{9}}{\left(3 n_{9}\right)^{2} R_{3}} \\
& R_{2}=\left(\frac{n_{7}}{2 n_{9}}\right)^{2}-\frac{2 n_{5}}{3 n_{9}}-R_{3} \\
& -\frac{n_{2}^{5}-3 n_{3} n_{7}+12 n_{1} n_{9}}{\left(3 n_{9}\right)^{2} R_{3}} \\
& R_{3}=\frac{R_{5}}{3 n_{9} \sqrt[3]{2\left(R_{4}+\sqrt{\left(R_{4}^{2}-4 R_{5}^{3}\right)}\right)}} \\
& R_{4}=2 n_{7}^{2}-9 n_{3} n_{5} n_{7}+27 n_{3}^{2} n_{9} \\
& +27 n_{7}^{2} n_{1}-72 n_{1} n_{5} n_{9} \\
& R_{1}=R_{2}-\frac{4 n_{5} n_{7} n_{9}-n_{7}^{3}-8 n_{3} n_{9}^{2}}{4 n_{9}^{3} \sqrt{R_{0}}} \\
& R_{5}=n_{5}^{2}-3 n_{3} n_{7}+12 n_{1} n_{9} \\
& b_{0}=\sqrt{-\frac{n_{1}}{n_{0} n_{9}}} \\
& b_{1}=\frac{\omega_{1}\left(\omega_{1}^{2}-\omega_{2}^{2}\right)\left(\omega_{1}^{2}-\omega_{3}^{2}\right)\left(\omega_{1}^{2}-\omega_{4}^{2}\right)}{Y_{4} \omega_{1}^{6}+Y_{3} \omega_{1}^{4}+Y_{2} \omega_{1}^{2}+Y_{1}} \\
& b_{2}=\frac{\omega_{2}\left(\omega_{2}^{2}-\omega_{1}^{2}\right)\left(\omega_{2}^{2}-\omega_{3}^{2}\right)\left(\omega_{2}^{2}-\omega_{4}^{2}\right)}{Y_{4} \omega_{2}^{6}+Y_{3} \omega_{2}^{4}+Y_{2} \omega_{2}^{2}+Y_{1}} \\
& b_{3}=\frac{\omega_{3}\left(\omega_{3}^{2}-\omega_{1}^{2}\right)\left(\omega_{3}^{2}-\omega_{2}^{2}\right)\left(\omega_{3}^{2}-\omega_{4}^{2}\right)}{Y_{4} \omega_{3}^{6}+Y_{3} \omega_{3}^{4}+Y_{2} \omega_{3}^{2}+Y_{1}} \\
& b_{4}=\frac{\omega_{4}\left(\omega_{4}^{2}-\omega_{1}^{2}\right)\left(\omega_{4}^{2}-\omega_{2}^{2}\right)\left(\omega_{4}^{2}-\omega_{3}^{2}\right)}{Y_{4} \omega_{4}^{6}+Y_{3} \omega_{4}^{4}+Y_{2} \omega_{4}^{2}+Y_{1}} \\
& Y_{1}=\frac{n_{2}-\frac{n_{0} n_{3}}{n_{1}}-\frac{n_{1}}{n_{9}}}{n_{9}} \\
& Y_{2}=\frac{n_{4}-\frac{n_{0} n_{5}}{n_{1}}-\frac{n_{3}}{n_{9}}}{n_{9}} \\
& Y_{3}=\frac{n_{6}-\frac{n_{0} n_{7}}{n_{1}}-\frac{n_{5}}{n_{9}}}{n_{9}} \\
& Y_{4}=\frac{n_{8}-\frac{n_{0} n_{9}}{n_{1}}-\frac{n_{7}}{n_{9}}}{n_{9}} .
\end{aligned}
$$

\section{ACKNOWLEDGMENT}

The authors would like to thank Christophe Guitton (University of Rennes 1, Rennes, France) and Gilles Picoult (INSA Rennes, Rennes) for the fabrication of the printed circuit board.

\section{REFERENCES}

[1] S. Holme, "Multiple passband filters for satellite applications," in Proc. 20th AIAA Int. Commun. Satell. Syst. Conf. Exhib., May 2002, p. 1993.

[2] M. Uhm, K. Ahn, I. Yom, and J. Kim, "A triple-passband waveguide filter with dual-mode resonators for ka band satellite applications," in Proc. 24th AIAA Int. Commun. Satell. Syst. Conf., Jun. 2006, p. 5450.

[3] J. Xu, W. Wu, and C. Miao, "Compact microstrip dual-/tri-/quad-band bandpass filter using open stubs loaded shorted stepped-impedance resonator," IEEE Trans. Microw. Theory Techn., vol. 61, no. 9, pp. 3187-3199, Sep. 2013.

[4] R. Gómez-García, J.-M. Munoz-Ferreras, and D. Psychogiou, "Split-type input-reflectionless multiband filters," IEEE Microw. Wireless Compon. Lett., vol. 28, no. 11, pp. 981-983, Nov. 2018.

[5] R. Gómez-García and A. C. Guyette, "Reconfigurable multi-band microwave filters," IEEE Trans. Microw. Theory Techn., vol. 63, no. 4 pp. 1294-1307, Apr. 2015.

[6] S.-J. Sun, T. Su, K. Deng, B. Wu, and C.-H. Liang, "Shorted-ended stepped-impedance dual-resonance resonator and its application to bandpass filters," IEEE Trans. Microw. Theory Techn., vol. 61, no. 9, pp. 3209-3215, Sep. 2013.

[7] R. Gomez-Garcia, L. Yang, and D. Psychogiou, "A frequency transformation for co-designed multi-passband/multi-embedded-notch RF filters," IEEE Trans. Circuits Syst. II, Exp. Brief, early access, Feb. 2, 2021, doi: 10.1109/TCSII.2021.3056584.

[8] L. Zhu, R. R. Mansour, and M. Yu, "Triple-band cavity bandpass filters," IEEE Trans. Microw. Theory Techn., vol. 66, no. 9, pp. 4057-4069, Sep. 2018.

[9] X. Guo, L. Zhu, and W. Wu, "Design method for multiband filters with compact configuration in substrate integrated waveguide," IEEE Trans. Microw. Theory Techn., vol. 66, no. 6, pp. 3011-3018, Jun. 2018.

[10] X.-P. Chen, K. Wu, and Z.-L. Li, "Dual-band and triple-band substrate integrated waveguide filters with chebyshev and quasi-elliptic responses," IEEE Trans. Microw. Theory Techn., vol. 55, no. 12, pp. 2569-2578, Dec. 2007.

[11] K. Zhou, C.-X. Zhou, and W. Wu, "Resonance characteristics of substrate-integrated rectangular cavity and their applications to dualband and wide-stopband bandpass filters design," IEEE Trans. Microw. Theory Techn., vol. 65, no. 5, pp. 1511-1524, May 2017.

[12] R. J. Cameron, "Advanced coupling matrix synthesis techniques for microwave filters," IEEE Trans. Microw. Theory Techn., vol. 51, no. 1, pp. 1-10, Jan. 2003.

[13] X. Shang, Y. Wang, G. Nicholson, and M. Lancaster, "Design of multiple-passband filters using coupling matrix optimisation," IET Microw., Antennas Propag., vol. 6, no. 1, pp. 24-30, Jan. 2012.

[14] M. Mokhtaari, J. Bornemann, K. Rambabu, and S. Amari, "Couplingmatrix design of dual and triple passband filters," IEEE Trans. Microw. Theory Techn., vol. 54, no. 11, pp. 3940-3946, Nov. 2006.

[15] S. Amari, "Synthesis of cross-coupled resonator filters using an analytical gradient-based optimization technique," IEEE Trans. Microw. Theory Techn., vol. 48, no. 9, pp. 1559-1564, Sep. 2000.

[16] Y. Zhang, K. A. Zaki, J. A. Ruiz-Cruz, and A. E. Atia, "Analytical synthesis of generalized multi-band microwave filters," in IEEE MTT-S Int. Microw. Symp. Dig., Jun. 2007, pp. 1273-1276.

[17] V. Crnojević-Bengin, Advances in Multi-Band Microstrip Filters. Cambridge, U.K.: Cambridge Univ. Press, 2015.

[18] J.-T. Kuo, T.-H. Yeh, and C.-C. Yeh, "Design of microstrip bandpass filters with a dual-passband response," IEEE Trans. Microw. Theory Techn., vol. 53, no. 4, pp. 1331-1337, Apr. 2005.

[19] J.-Y. Wu and W.-H. Tu, "Design of quad-band bandpass filter with multiple transmission zeros," Electron. Lett., vol. 47, no. 8, pp. 502-503, Apr. 2011.

[20] W.-Y. Chen, M.-H. Weng, and S.-J. Chang, "A new tri-band bandpass filter based on stub-loaded step-impedance resonator," IEEE Microw. Wireless Compon. Lett., vol. 22, no. 4, pp. 179-181, Apr. 2012.

[21] C.-F. Chen, "Design of a compact microstrip quint-band filter based on the tri-mode stub-loaded stepped-impedance resonators," IEEE Microw. Wireless Compon. Lett., vol. 22, no. 7, pp. 357-359, Jul. 2012.

[22] J.-S. G. Hong and M. J. Lancaster, Microstrip Filters for RF/Microwave Applications, 2nd ed. New York, NY, USA: Wiley, 2011.

[23] K.-W. Hsu, J.-H. Lin, and W.-H. Tu, "Compact sext-band bandpass filter with sharp rejection response," IEEE Microw. Wireless Compon. Lett., vol. 24, no. 9, pp. 593-595, Sep. 2014.

[24] W.-H. Tu and K.-W. Hsu, "Design of sext-band bandpass filter and sextaplexer using semilumped resonators for system in a package," IEEE Trans. Compon., Package Manuf. Technol., vol. 5, no. 2, pp. 265-273, Feb. 2015.

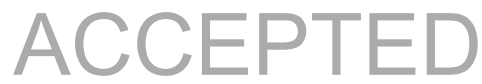


[25] C. Quendo, E. Rius, and C. Person, "Narrow bandpass filters using dualbehavior resonators," IEEE Trans. Microw. Theory Techn., vol. 51, no. 3, pp. 734-743, Mar. 2003.

[26] C. Quendo et al., "Planar tri-band filter based on dual behavior resonator (DBR)," in Proc. Eur. Microw. Conf., Oct. 2005, pp. 269-272.

[27] G. Macchiarella and S. Tamiazzo, "Design techniques for dualpassband filters," IEEE Trans. Microw. Theory Techn., vol. 53, no. 11, pp. 3265-3271, Nov. 2005.

[28] X. Guan, Z. Ma, P. Cai, Y. Kobayashi, T. Anada, and G. Hagiwara, "Synthesis of dual-band bandpass filters using successive frequency transformations and circuit conversions," IEEE Microw. Wireless Compon. Lett., vol. 16, no. 3, pp. 110-112, Mar. 2006.

[29] H. Di, B. Wu, X. Lai, and C.-H. Liang, "Synthesis of cross-coupled triple-passband filters based on frequency transformation," IEEE Microw. Wireless Compon. Lett., vol. 20, no. 8, pp. 432-434, Aug. 2010.

[30] P. Ma et al., "A design method of multimode multiband bandpass filters," IEEE Trans. Microw. Theory Techn., vol. 66, no. 6, pp. 2791-2799, Jun. 2018.

[31] A. García-Lampérez and M. Salazar-Palma, "Single-band to multiband frequency transformation for multiband filters," IEEE Trans. Microw. Theory Techn., vol. 59, no. 12, pp. 3048-3058, Dec. 2011.

[32] G. L. Matthaei, L. Young, and E. M. T. Jones, Microwave Filters, Impedance-Matching Networks, and Coupling Structures. Dedham, MA, USA: Artech House, 1980.

[33] Y. Wu, E. Fourn, P. Besnier, and C. Quendo, "Direct synthesis of quadband band-pass filter by frequency transformation methods," in Proc. 49th Eur. Microw. Conf. (EuMC), Oct. 2019, pp. 196-199.

[34] A. Manchec, Y. Clavet, C. Quendo, E. Rius, J. F. Favennec, and C. Person, "Cross-coupled microstrip dual behavior resonator (DBR) filter," in Proc. Eur. Microw. Conf., Sep. 2006, pp. 556-559.

[35] J.-C. Lu, Y.-W. Lin, C.-K. Liao, and C.-Y. Chang, "Five-pole parallelcoupled microstrip cascade quadruplet filters for high selectivity and flat group delay," in Proc. Asia Pacific Microw. Conf., Dec. 2009, pp. $878-881$.

[36] R. J. Cameron, C. M. Kudsia, and R. R. Mansour, Microwave Filters for Communication Systems: Fundamentals, Design, and Applications, 2nd ed. Hoboken, NJ, USA: Wiley, 2018.

[37] Z.-C. Guo, L. Zhu, and S.-W. Wong, "A quantitative approach for direct synthesis of bandpass filters composed of transversal resonators," IEEE Trans. Circuits Syst. II, Exp. Briefs, vol. 66, no. 4, pp. 577-581, Apr. 2019.

[38] R. J. Cameron, "General coupling matrix synthesis methods for chebyshev filtering functions," IEEE Trans. Microw. Theory Techn., vol. 47, no. 4, pp. 433-442, Apr. 1999.

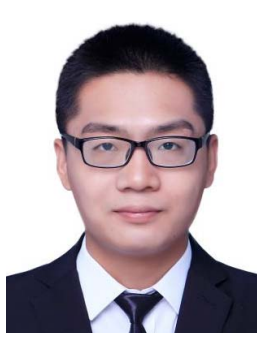

Yi Wu (Student Member, IEEE) was born in Tai' an, Shandong, China. He is currently pursuing the Ph.D. degree at the National Institute of Applied Sciences (INSA Rennes), Rennes, France.

His research interests include the design of filters for microwave applications and the optical instruments for space applications.

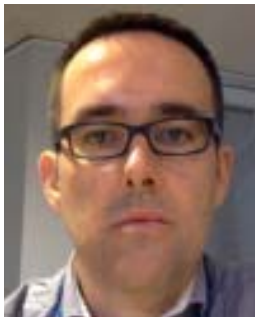

Erwan Fourn (Member, IEEE) received the master's and $\mathrm{Ph} . \mathrm{D}$. degrees in electronics from the University of Brest, Brest, France, in 2001 and 2004, respectively.

He was a Post-Doctoral Fellow with the Laboratory for Analysis and Architecture of Systems (LAAS-CNRS), Toulouse, France. He has been the Co-Director of the joint-lab MERLIN between the Institut d'Électronique et des Technologies du numéRiques (IETR), Rennes, France, and Thales Alenia Space, Cannes, France, since 2020. He is currently an Associate Professor with the National Institute of Applied Sciences (INSA Rennes), Rennes. He is within IETR, where his research interests include the design of directive antennas (reflect-array and transmit-array or FSS) in one side and bandpass filters in the other side both mainly for multiband and/or reconfigurable applications at microwave and millimeter wave frequencies.

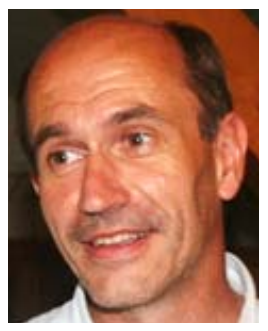

Philippe Besnier (Senior Member, IEEE) received the diplôme d'ingénieur degree from the École Universitaire D’Ingénieurs de Lille (EUDIL), Lille, France, in 1990, and the Ph.D. degree in electronics from the University of Lille, Lille, in 1993.

Following a one-year period at the Office National d'Etudes et de Recherches Aérospatiales (ONERA), Meudon, Meudon, France, as an Assistant Scientist with the Electromagnetic Compatibility (EMC) Division, he was with the Laboratory of Radio-Propagation and Electronics (LRPE), University of Lille, as a Researcher with the Centre National de la Recherche Scientifique (CNRS), Paris, France, from 1994 to 1997. From 1997 to 2002, he was the Director of the Centre d'Etudes et de Recherches en Protection Electromagnétique (CERPEM): a not-for-profit organization for research, expertise, and training in EMC and related activities, based in Laval, France. He also co-founded TEKCEM in 1998, a small business company specialized in turn-key systems for EMC measurements. Back to CNRS in 2002, he has been since then with the Institut d'Electronique et des Technologies du numéRique (IETR), Rennes, France. He was appointed as the CNRS Senior Researcher (directeur de recherche) in 2013. He was the Co-Head of the "Antennas and Microwave Devices" Research Department, IETR, from 2012 to 2016. He headed the WAVES (electromagnetic waves in complex media) team during the first semester of 2017. Since July 2017, he has been the Deputy Director of IETR. His research interests include interference analysis on cable harnesses (including electromagnetic topology), theory and application of reverberation chambers, shielding and absorbing techniques, near-field probing, and uncertainty quantification in EMC modeling.

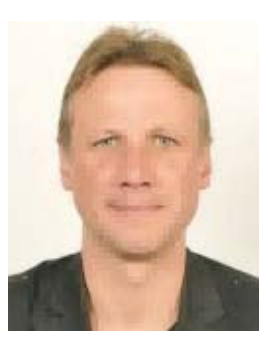

Cédric Quendo (Senior Member, IEEE) received the Electrical Engineer degree and Ph.D. degree in electrical engineering from the University of Brest, Brest, France, in 1999 and 2001, respectively.

From 2001 to 2010, he gave courses and conducted research in several institutes. Since 2010, he has been a Professor with the Electronic Department, University of Brest. From 2012 to 2016, he was the Vice President of the University of Brest. From 2017 to 2020, he was the Head of the Microwaves Optoelectronics and Materials Group, Lab-STICC Laboratory (more than 100 persons), Brest. He is currently the Head of Lateral a joint Laboratory with Thales LAS-OME, Elancourt, France. His research interests include the modeling and design of microwave devices for microwave and millimeter-wave applications. 